\title{
Grand challenges for colloidal materials and interfaces: dancing on nano-stage
}

\author{
Jie-Sheng Chen* \\ School of Chemistry and Chemical Engineering, Shanghai Jiao Tong University, Shanghai, China \\ *Correspondence: chemcj@sjtu.edu.cn \\ Edited and reviewed by: \\ Guang-Ling Song, Oak Ridge National Laboratory, USA
}

Keywords: colloids, interfaces, nanoscale, assembly, biomimicry

Colloids are ubiquitous, and the field of Colloidal Materials and Interfaces is an everrenewing area rooted in a long-standing tradition (Thomas, 1833; Sanders et al., 1964; Whitesides and Grzybowski, 2002; Jaakko et al., 2013). The field lies at the interdisciplinary intersection of branches including chemistry, physics, biology, engineering, nanoscience, materials, medicine, and food science. Classical colloids consist of tiny structures with at least one dimension falling within the $1-1000 \mathrm{~nm}$ range; this can be further extended to 10,000 to $100,000 \mathrm{~nm}$ in applied colloid science. Though colloidal dispersions have been studied for more than 200 years, we face new questions with the revolution of characterization techniques and with our better understanding of the real nature of colloidal materials and interfaces. While interfacial properties for conventional mineral or polymer colloids of the micrometer scale are well elucidated, the nanometer range remains to be explored.

One general challenge in colloid and interface science is to precisely control the size and shape of nanocolloid particles. Current chemical methods supply excellent control in the morphology of ligand-protected nanocrystals, nevertheless, they can only be considered as organic-inorganic hybrid nanocolloids (Alivisatos, 1996). In case of less-protected nanocolloids, the stability and specific physicochemical properties should be well balanced. With bare surface and controlled morphology, these may prove to be useful for practical applications. Further precise control of the dopants, core-shell structures, and/or the crystalline facets (Norris et al., 2008) may result in functional nanocolloids with novel or enhanced properties.
The second grand challenge is the assembly of nanocolloids into hierarchical structures and thin films in solution and at various interfaces, e.g., air-liquid interfaces, solid-liquid interfaces, and also water-oil interfaces (Li et al., 2006). The rational selection of surface ligands or stabilizers becomes a powerful tool to tune interfacial interaction between the colloid components and/or the dispersion medium. Besides the assembly of nanoparticles, organic ligands, and polymer molecules, the assembly of ions and clusters is highly challenging and it is to be exploited to fabricate uniform thin films with novel functions (Abellán et al., 2011). Since the interactions between these components and substrates are sensitive to the chemical environment and temperature, they are fundamental factors for the formation of high-quality thin films. Fabrication of large-area thin films would require a homogenous chemical environment with a decreased temperature gradient over long distances. Lack of reproductive methods for preparing functional thin films in large area is still the main hurdle to be addressed for their practical applications.

Plenty of opportunities remain to develop novel functions of colloidal materials since the interface effects such as the ligand and Mott-Schottky effects (Li and Antonietti, 2013) on materials functions have not yet been well investigated. Taking catalysis as an example of practical applications of nanoparticles, metal nanoparticles dispersed on various supports form the basis of important catalysts for chemical industry and future energy supply where both the nanocolloids and interfaces are involved. Physicochemical properties of nanoparticles and interface metal-support effect exert a dominant effect on the final catalytic performance.
For medical and biotechnological applications, fabricating nanodrops and nanobubbles and their delivery remain in focus. Microfluidic setups or nano-motors for certain targets of microscale reactions and characterization for analysis and therapy are the main points to be addressed since the development of nanomedical applications has become a priority of current medical studies, such as cancer detection and therapy. In order to achieve this, physical properties of intracellular structures and the interactions between cells and nanoparticles need to be better studied. More efforts should be focused on these objectives to further initiate the real applications of nanocolloids in disease-fighting (Cho et al., 2008).

For solving complex human problems, construction of functional bio-colloids and assemblies mimicking the structure, function, and manner of natural beings have been extensively explored for decades. Nevertheless, nano biomimicry, which imitates nanoscale structures and processes, or follows the basic principles, still remains in its infancy. Moreover, a great variety of nanostructures in nature supply us sustainable templates for largescale synthesis of nanoparticles, nanotubes, nanowires, and even uniform nano-arrays in low cost (Yao et al., 2013). These "bionanomaterials" have wide applications in catalysis, energy storage, filtration, photovoltaics, sensors, and medical uses. The efficiency of separating these useful biotemplates from their natural mother compounds is still low.

With respect to the practical use of nanocolloids, toxicity of nanocolloids requires intensive investigation (Elsaesser and Howard, 2012; Rivera-Gil et al., 2013). Nanoparticles can penetrate the skin and even the cells themselves. The 
high surficial energy and the unique size-dependent physicochemical properties make nanocolloids highly active to form specific species and thus lead to unexpected effects on human health and the environment. Despite the fact that cytotoxicity of certain nanocolloids has not been elucidated yet, the presence of tiny particles in both air and water already affect our daily life (Prathe, 2009). Understanding the transfer paths and manners of these nanoparticles is highly important for safe use of nanocolloid-based products and for removal of unwanted nanocolloids from polluted air and water.

I just listed some representative and special challenges for colloidal materials and interfaces. Indeed, the highly dynamic nature of this fast-evolving field makes it impossible to cover all aspects of research in the field. Nevertheless, gradually, renewable technologies in the realm of colloidal materials and interfaces with contributions from researchers in chemistry, physics, chemical engineering, and materials science, will supply us more powerful tools to engineer the functions of nanocolloids and their interfaces for specific tasks. This will lead to better materials, more efficient catalysts, improved health care, and a cleaner world.

\section{REFERENCES}

Abellán, P., Moreno, C., Sandiumenge, F., Obradors, X. and Casanove, M. J. (2011). Misfit relaxation of $\mathrm{La}_{0 .}{ }_{7} \mathrm{Sr}_{0.3} \mathrm{MnO}_{3}$ thin films by a nanodot segregation mechanism. Appl. Phys. Lett. 98, 041903. doi:10.1063/1.3549182

Alivisatos, A. P. (1996). Semiconductor clusters, nanocrystals, and quantum dots. Science 271 , 933-937. doi:10.1126/science.271.5251.933

Cho, K., Wang, X., Nie, S. M., Chen, Z., and Shin, D. M. (2008). Therapeutic nanoparticles for drug delivery in cancer. Clin. Cancer Res. 14, 1310-1316. doi:10.1158/1078-0432.CCR-07-1441

Elsaesser, A., and Howard, C. V. (2012). Toxicology of nanoparticles. Adv. Drug Deliv. Rev. 64, 129-137. doi:10.1016/j.addr.2011.09.001

Jaakko, V. I. T., Mika, L., Ludwik, L., Robin, H. A. R., and Olli, I. (2013). Switchable static and dynamic selfassembly of magnetic droplets on superhydrophobic surfaces. Science 341, 253-257. doi:10.1126/ science. 1233775

Li, X.-H., and Antonietti, M. (2013). Metal nanoparticles at mesoporous $\mathrm{N}$-doped carbons and carbon nitrides: functional Mott-Schottky heterojunctions for catalysis. Chem. Soc. Rev. 42, 6593-6604. doi:10. 1039/C3CS60067J

Li, X.-H., Zhang, D.-H., and Chen, J.-S. (2006). Synthesis of amphiphilic superparamagnetic ferrite/block copolymer hollow submicrospheres. J. Am. Chem. Soc. 128, 8382-8383. doi:10.1021/ja061460g

Norris, D. J., Efros, A. L., and Erwin, S. C. (2008). Doped nanocrystals. Science 319, 1776-1779. doi: 10.1126/science. 1143802

Prathe, B. (2009). Control of Airborne Particles. Available at: http://ohsonline.com/articles/2009/10/01/ control-of-airborne-particles.aspx?admgarea $=$ ht. IndoorAirQuality
Rivera-Gil, P., Jimenez de Aberasturi, D., Wulf, V., Pelaz, B., Pino, P. D., Zhao, Y., et al. (2013). The challenge to relate the physicochemical properties of colloidal nanoparticles to their cytotoxicity. Acc. Chem. Res. 46, 743-749. doi:10.1021/ar300039j

Sanders, J. V., Sanders, J. V., and Segnit, E. R. (1964). Structure of opal. Nature 204, 1151. doi:10.1038/ 204990a0

Thomas, G. (1833). Researches on the arsenates, phosphates, and modifications of phosphoric acid. Philos. Trans. 123, 253-284. doi:10.1098/rstl.1833.0015

Whitesides, G. M., and Grzybowski, B. (2002). Selfassembly at all scales. Science 295, 2418-2421. doi:10.1126/science.1070821

Yao, H., Zheng, G., Li, W., McDowell, M. T., Seh, Z., Liu, N., et al. (2013). Crab shells as sustainable templates from nature for nanostructured battery electrodes. Nano Lett. 13, 3385-3389. doi:10.1021/ nl401729r

Received: 30 January 2014; accepted: 13 February 2014; published online: 20 February 2014.

Citation: Chen J-S (2014) Grand challenges for colloidal materials and interfaces: dancing on nano-stage. Front. Mater. 1:1. doi:10.3389/fmats.2014.00001

This article was submitted to Colloidal Materials and Interfaces, a section of the journal Frontiers in Materials. Copyright (c) 2014 Chen. This is an open-access article distributed under the terms of the Creative Commons Attribution License (CC BY). The use, distribution or reproduction in other forums is permitted, provided the original author(s) or licensor are credited and that the original publication in this journal is cited, in accordance with accepted academic practice. No use, distribution or reproduction is permitted which does not comply with these terms. 